\title{
Sharp bounds on the radius of relativistic charged spheres: Guilfoyle's stars saturate the Buchdahl-Andréasson bound
}

\author{
José P. S. Lemos \\ Centro Multidisciplinar de Astrofísica - CENTRA, Departamento de Física, Instituto \\ Superior Técnico - IST, Universidade de Lisboa - UL, Avenida Rovisco Pais 1, \\ 1049-001 Lisboa, Portugal \\ Email: joselemos@ist.utl.pt
}

Vilson T. Zanchin

Centro de Ciências Naturais e Humanas, Universidade Federal do ABC, Avenida dos Estados 5001, 09210-580 Santo André, São Paulo, Brazil

Email: zanchin@ufabc.edu.br

\begin{abstract}
.
Buchdahl, by imposing a few reasonable physical assumptions on the matter, i.e., its density is a nonincreasing function of the radius and the fluid is a perfect fluid, and on the configuration, such as the exterior is the Schwarzschild solution, found that the radius $r_{0}$ to mass $m$ ratio of a star would obey the bound $r_{0} / m \geq 9 / 4$, the Buchdahl bound. He also noted that the bound was saturated by the Schwarzschild interior solution, i.e., the solution with $\rho_{\mathrm{m}}(r)=$ constant, where $\rho_{\mathrm{m}}(r)$ is the energy density of the matter at $r$, when the central central pressure blows to infinity. Generalizations of this bound in various forms have been studied. An important generalization was given by Andréasson by including electrically charged matter and imposing a different set of conditions, namely, $p+2 p_{T} \leq \rho_{\mathrm{m}}$, where $p$ is the radial pressure and $p_{T}$ the tangential pressure. His bound is sharp and is given by $r_{0} / m \geq 9 /\left(1+\sqrt{1+3 q^{2} / r_{0}^{2}}\right)^{2}$, the Buchdahl-Andréasson bound, with $q$ being the total electric charge of the star. For $q=0$ one recovers the Buchdahl bound. However, following Andréasson's proof, the configuration that saturates the Buchdahl bound is an uncharged shell, rather than the Schwarzschild interior solution. By extension, the configurations that saturate the electrically charged Buchdahl-Andréasson bound are charged shells. One could expect then, in turn, that there should exist an electrically charged equivalent to the interior Schwarzschild limit. We find here that this equivalent is provided by the equation $\rho_{\mathrm{m}}(r)+Q^{2}(r) /\left(8 \pi r^{4}\right)=$ constant, where $Q(r)$ is the electric charge at $r$. This equation was put forward by Cooperstock and de la Cruz, and Florides, and realized in Guilfoyle's stars. When the central pressure goes to infinity Guilfoyle's stars are configurations that also saturate the Buchdahl-Andréasson bound. It remains to find a proof in Buchdahl's manner such that these configurations are the limiting configurations of the bound.
\end{abstract}




\section{Introduction}

Schwarzschild was the first to put forward the idea of a compact object [1]. He found an exact solution in general relativity for a spherical star whose matter content is made of an incompressible fluid, i.e., $\rho_{\mathrm{m}}(r)=$ constant, where $\rho_{\mathrm{m}}(r)$ is the energy density at radius $r$. This solution is called the interior Schwarzschild solution. Moreover he also established a compactness limit for these configurations, the interior Schwarzschild limit. For a star of radius $r_{0}$ and mass $m$, the central pressure $p_{c}$ of the interior Schwarzschild solution becomes infinite when $r_{0} / m=9 / 4$ (we use units such that $G=1$ and $c=1$, where $G$ is Newton constant of gravitation and $c$ is the speed of the light.). Thus, for smaller $r_{0}$ there is certainly no fluid pressure capable to sustain the configuration against the gravitational pull, and it will collapse.

The existence of an upper bound for the compactness of the matter within a star in equilibrium shown by Schwarzschild [1] gave rise to further interest on the subject. Indeed, Buchdahl [2] analyzed a spherically symmetric distribution of matter in a model-independent manner, in quite general terms. He imposed a few physically reasonable restrictions, namely, that the fluid's energy density $\rho_{\mathrm{m}}$ is non-negative and non-increasing outward, the pressure $p$ is non-negative and isotropic, i.e., the fluid is perfect, and the boundary defined by $p=0$ is matched to the exterior Schwarzschild solution. He fount that there was a compactness bound for the configuration given by $r_{0} / m \geq 9 / 4$. In his proof it was clear that the limiting interior Schwarzschild solution (i.e., $\rho=$ constant and $p_{c} \rightarrow \infty$ ) is the configuration that saturates the bound.

A different proof for Buchdahl's inequality was given by Andréasson [3]. He assumed a different set of restrictions, namely, that $p+2 p_{T} \leq \rho_{\mathrm{m}}$, where $p$ is now the radial pressure, and $p_{T}$ is the tangential pressure, with all three quantities being positive, and the boundary defined by $p=0$ is matched to the exterior Schwarzschild solution. With this set of assumptions it was also found that the compactness bound is Buchdahl's, $r_{0} / m \geq 9 / 4$. However, rather than having the limiting interior Schwarzschild solution as saturating the bound, Andréasson found that the bound was saturated by a compact thin shell. Indeed, since a thin shell has by definition $p=0$, a simple exercise shows that for a thin shell with $2 p_{T}=\rho_{\mathrm{m}}$ one finds $r_{0} / m=9 / 4$. Still assuming $p+2 p_{T} \leq \rho_{\mathrm{m}}$ a proof of Buchdahl's bound, different from [3] was given by Stalker and Karageorgis [4].

In order to obtain still lower limits for the bound one has to resort to matter with extra properties. The ultimate goal is perhaps to have a configuration hovering at its own gravitational radius. One form of matter that yields repulsion is electrically charged matter. Thus, with new compactness limits in mind, Andréasson sought to devise a bound, the Buchdahl-Andréasson bound for electrically charged matter [5]. He assumed still that $p+2 p_{T} \leq \rho_{\mathrm{m}}$, and that the boundary is matched to the exterior ReissnerNordström solution. Relying on Stalker and Karageorgis's proof [4], Andréasson found the bound $r_{0} / m \geq 9 /\left(1+\sqrt{1+3 q^{2} / r_{0}^{2}}\right)^{2}$, where $q$ is the total electric charge of the 
star. Note that generically all the configurations that satisfy the Buchdahl-Andréasson bound are above the gravitational radius $r_{+}$of the star given by $r_{+}=m+\sqrt{m^{2}-q^{2}}$. For $q=0$ the Buchdahl bound $r_{0} / m \geq 9 / 4$ follows immediately, which yields an $r_{0}$ greater than the gravitational radius $r_{+}=2 m$. For $q=r_{0}$ one obtains $r_{0} / m \geq 1$, whose lower bound $r_{0}=m$ is a configuration at its own gravitational radius $r_{+}$, indeed in this extreme case $r_{0}=r_{+}=m=q$. This latter configuration is a quasiblack hole. Following the previous proof for the uncharged case [3, 4], it was found [5] that the bound is saturated by the compact thin shells that obey $2 p_{T}=\rho_{\mathrm{m}}$. These shells are such that $r_{0} / m=9 /\left(1+\sqrt{1+3 q^{2} / r_{0}^{2}}\right)^{2}$.

A natural question, raised legitimately by Andréasson [5], is what, if any, are the electrically charged configurations that saturate the bound and that correspond to the (uncharged) interior Schwarzschild limit configuration. In other words, are there electrically charged configurations, other than thin shells, that saturate the bound? And if yes, do they correspond in the uncharged case to the interior Schwarzschild limit configuration?

Here we are able to answer these questions in the positive. Defining $\rho_{\mathrm{m}}(r)$ as the energy density of the matter at $r$ and $Q(r)$ as the electric charge at $r$, we show that the equation of state suggested by Cooperstock and de la Cruz [6] and Florides [7], namely, $\rho_{\mathrm{m}}(r)+Q^{2}(r) /\left(8 \pi r^{4}\right)=$ constant, allows to build stars [8, 9], Guilfoyle's stars, which for $p_{c} \rightarrow \infty$, saturate the Buchdahl-Andréasson bound. This is not a complete surprise. Cooperstock and de la Cruz [6] and Florides [7] argue forcefully that the above equation of state is the electric charge equivalent to the incompressible equation of state set by Schwarzschild for the interior solution. Given that these electrically charged configurations are the analogous to the interior Schwarzschild configurations, this suggests that a proof of the Buchdahl-Andréasson bound along the lines of Buchdahl [2], rather than along the lines of Andréasson [5], should be in sight. Of course, other electrically charged fluids do not obey the Buchdahl-Andréasson bound. It was shown in [10, 11] that a fluid with $\rho_{\mathrm{m}}(r)=$ contant and with a stiff electrically charged distribution $Q(r)$, different from the Cooperstock-de la Cruz-Florides distribution, does not saturate the Buchdahl-Andréasson bound, although it comes close to.

The present paper is organized as follows. In Sec. 2 we write the particular solution of Guilfoyle's work that interests us here, putting the relevant relations in the appropriate form for further analysis. Section 3 is dedicated to find the electrical interior Schwarzschild limit of the Guilfoyle solution. We take the central pressure to infinity and obtain a constraint among the parameters of the solution. We show that this constraint saturates the Buchdahl-Andréasson bound. In Sec. 4 we conclude. 


\section{The Guilfoyle solutions}

The Guilfoyle star solutions [8] are exact equilibrium solutions of Einstein-Maxwell equations that were put forward to represent electrically charged stars. The metric is conveniently written in the spherically symmetric form, namely,

$$
d s^{2}=-B(r) d t^{2}+A(r) d r^{2}+r^{2}\left(d \theta^{2}+\sin ^{2} \theta d \varphi^{2}\right) .
$$

where $t, r, \theta, \varphi$, are the usual Schwarzschild coordinates, and the metric functions $A(r)$ and $B(r)$ depend on the radial coordinate $r$ only.

The stars are made of a cold electrically charged fluid with pressure, have a spherical surface of radius at $r=r_{0}$, the star's surface, where the matter pressure vanishes, and exterior to that, for $r>r_{0}$, the spacetime is the electromagnetic vacuum, whose metric functions $A(r)$ and $B(r)$, and electric potential $\phi(r)$, are given by the ReissnerNordström solution

$$
\begin{aligned}
& B(r)=A^{-1}(r)=1-\frac{2 m}{r}+\frac{q^{2}}{r^{2}}, \\
& \phi(r)=\frac{q}{r}+\text { const. }
\end{aligned}
$$

with $m$ and $q$ being respectively the total mass and total charge of the stars. One of the zeros of the metric functions of equation (2) gives the gravitational radius $r_{+}$, i.e.,

$$
r_{+}=m+\sqrt{m^{2}-q^{2}} .
$$

The gravitational radius $r_{+}$is the horizon radius if the solution is a vacuum solution. The other zero of the metric functions of equation (2) is the Cauchy horizon radius $r_{-}$, given by $r_{-}=m-\sqrt{m^{2}-q^{2}}$. It will not be mentioned further as it will not be needed in our analysis.

The systems we are interested here belong to a particular class of the Guilfoyle solutions. The interior of these solutions is characterized by the fact that the metric potential $B(r)$ and the electric potential $\phi(r)$ are functionally related through a WeylGuilfoyle relation, namely, $B(r)=a[-\epsilon \phi(r)+b]^{2}$, with $a$ and $b$ being arbitrary constants and $\epsilon= \pm 1$. We choose the solutions for which $b=0$. Another interesting feature of these charged fluids is the relation between the energy density $\rho_{\mathrm{m}}$, the pressure $p$, the charge density $\rho_{\mathrm{e}}$, and the electromagnetic energy density $\rho_{\mathrm{em}}$ defined by $\rho_{\mathrm{em}}=\left(\nabla_{i} \phi\right)^{2} / 8 \pi B$. All these quantities satisfy the constraint $\sqrt{a} \rho_{\mathrm{e}}=$ $\epsilon\left[\rho_{\mathrm{m}}+3 p+(1-a) \rho_{\mathrm{em}}\right]$.

The total electric charge within a sphere of radius $r, Q(r)$, which follows by integrating the only nonzero component of Maxwell equations, may be written as $Q(r)=\frac{\phi^{\prime}(r) r^{2}}{\sqrt{B(r) A(r)}}$, where the prime denotes the derivative with respect to the radial coordinate $r$ and without loss of generality an integration constant was set to zero. The class of objects we are interested in here obey $\epsilon \phi(r)=-\sqrt{B(r) / a}$. After such 
a relation, the electric charge inside a spherical surface of radius $r$ is now given by $Q(r)=-\epsilon r^{2} B^{\prime}(r) /(2 \sqrt{a A(r)} B(r))$. The Guilfoyle's ansatz is completed by the assumption that the total energy density, i.e., $\rho_{\mathrm{m}}(r)+\rho_{\mathrm{em}}(r)=\rho_{\mathrm{m}}(r)+Q^{2}(r) /\left(8 \pi r^{4}\right)$, is a constant, namely,

$$
\rho_{\mathrm{m}}(r)+\frac{Q^{2}(r)}{8 \pi r^{4}}=\frac{3}{R^{2}} .
$$

with $R$ being a free parameter of the model. Equation (5) was devised by Cooperstock and de la Cruz [6] and Florides [7]. With this, Einstein-Maxwell equations furnish the metric potentials $A(r)$ and $B(r)$,

$$
\begin{aligned}
& A(r)=\left(1-\frac{r^{2}}{R^{2}}\right)^{-1}, \\
& B(r)=\left[\frac{2-a}{a}\left(k_{0} \sqrt{1-\frac{r^{2}}{R^{2}}}-k_{1}\right)\right]^{2 a /(a-2)},
\end{aligned}
$$

and the electric charge $Q(r)$, and the fluid quantities $\rho_{\mathrm{m}}(r)$ and $p(r)$,

$$
\begin{aligned}
& Q(r)=\frac{\epsilon \sqrt{a}}{2-a} \frac{k_{0} r^{3}}{R^{2}}\left(k_{0} \sqrt{1-\frac{r^{2}}{R^{2}}}-k_{1}\right)^{-1}, \\
& 8 \pi \rho_{\mathrm{m}}(r)=\frac{3}{R^{2}}-\frac{Q^{2}(r)}{r^{4}}, \\
& 8 \pi p(r)=-\frac{1}{R^{2}}+\frac{Q^{2}(r)}{r^{4}}+\frac{2 \varepsilon Q(r)}{r^{3}} \sqrt{1-\frac{r^{2}}{R^{2}}},
\end{aligned}
$$

with $k_{0}$ and $k_{1}$ being integration constants.

There are four constraints. The continuity of the metric functions on the surface $r=r_{0}$ yields two constraints. Another constraint comes from the continuity of the first derivative of the metric function $B(r)$ across the boundary surface $r=r_{0}$, implying that the pressure vanishes at the boundary. An additional constraint comes from the fact that the solution must be of Weyl-Guilfoyle type, i.e., here satisfying the relation $B(r)=a \phi^{2}(r)$ throughout the spacetime, even at the boundary. The imposition of smooth boundary conditions on the boundary surface $r=r_{0}$, gives the two constraints that determine the two integrations constants $k_{0}$ and $k_{1}$, related then to the parameters $m, q$, and $R$, and $r_{0}$ by

$$
\begin{aligned}
& k_{0}=\frac{R^{2}}{r_{0}^{2}}\left(\frac{m}{r_{0}}-\frac{q^{2}}{r_{0}^{2}}\right)\left(1-\frac{r_{0}^{2}}{R^{2}}\right)^{-1 / a}, \\
& k_{1}=k_{0} \sqrt{1-\frac{r_{0}^{2}}{R^{2}}}\left[1-\frac{a r_{0}^{2}}{2-a R^{2}}\left(\frac{m}{r_{0}}-\frac{q^{2}}{r_{0}^{2}}\right)^{-1}\right] .
\end{aligned}
$$

The other two constraints give the following relations

$$
\begin{aligned}
& \frac{m}{r_{0}}=\frac{1}{2}\left(\frac{r_{0}^{2}}{R^{2}}+\frac{q^{2}}{r_{0}^{2}}\right), \\
& \frac{m}{q}=(1-a) \frac{q}{r_{0}}+\sqrt{a\left[1+(a-1) \frac{q^{2}}{r_{0}^{2}}\right]} .
\end{aligned}
$$


Hence, from the several parameters of the model, $m, q, a, r_{0}$, and $R$, three of them remain free.

There are additional constraints. We impose the stars are not overcharged, which implies that the pressure is non-negative. This implies also

$$
a \geq 1 \text {. }
$$

Furthermore we impose there are no trapped surfaces, i.e.,

$$
r_{0} \geq r_{+}
$$

where we allow for the equality, and $r_{+}$is the gravitational radius of the configuration given in equation (44). Now, by assumption, the stars are not overcharged so that $q / m \leq 1$ and thus

$$
\frac{r_{0}}{q} \geq 1, \quad \frac{r_{0}}{m} \geq 1
$$

are the constraints.

In the following we analyze the infinite central pressure limit of these compact stars, and obtain configurations that saturate the Buchdahl-Andréasson bound.

\section{The electrical interior Schwarzschild limit and the Buchdahl-Andréasson bound}

As we know from the case of the interior Schwarzschild solution, the lower bound of the ratio $r_{0} / m$ of the Schwarzschild stars is found by allowing the central pressure to assume indefinitely high values. Hence, we search for the range of parameters in Guilfoyle's solutions for which the central pressure reaches indefinitely large values. The pressure $p$ given in equation (10) behaves for small $r$ as $p(r) \sim \frac{r^{3}}{k_{0}-k_{1}}$. Thus, the pressure is in general well behaved at $r=0$ unless $k_{0}-k_{1}=0$. Therefore, the central pressure tends to infinity, $p_{c} \rightarrow \infty$, when

$$
k_{0}=k_{1} .
$$

Using relations (11), (12), and (13), equation (18) yields

$$
\sqrt{1-\frac{r_{0}^{2}}{R^{2}}}\left[1-\frac{2 a}{2-a} \frac{r_{0}^{2}}{R^{2}}\left(\frac{r_{0}^{2}}{R^{2}}-\frac{q^{2}}{r_{0}^{2}}\right)^{-1}\right]=1 .
$$

Solving for $a$ equation (14), substituting it into the last equation, and then solving for $q^{2} / R^{2}$ we find two solutions. One of them yields values of charge whose respective Guilfoyle solutions are in a range of parameters which do not correspond to charged stars. Namely, they are black holes with infinite central pressure. The second solution is the one that has interest in the present context, and is given by

$$
\frac{q^{2}}{r_{0}^{2}}=4\left(1-\sqrt{1-\frac{r_{0}^{2}}{R^{2}}}\right)-3 \frac{r_{0}^{2}}{R^{2}}
$$


Equation (13) can be written for $\frac{r_{0}^{2}}{R^{2}}$, i.e., $\frac{r_{0}^{2}}{R^{2}}=\frac{2 m}{r_{0}}-\frac{q^{2}}{r_{0}^{2}}$, which upon inserting in equation (20) gives the radius to mass ratio as a function of the electric charge for the electrical interior Schwarzschild limit, namely, $\frac{r_{0}}{m}=\left[\frac{2}{9}\left(1+\sqrt{1+\frac{3 q^{2}}{r_{0}^{2}}}\right)+\frac{q^{2}}{3 r_{0}^{2}}\right]^{-1}$, or equivalently,

$$
\frac{r_{0}}{m}=\frac{1}{\left(\frac{1}{3}+\sqrt{\frac{1}{9}+\frac{q^{2}}{3 r_{0}^{2}}}\right)^{2}},
$$

which is the upper limit of compactness for these stars.

Consider, now the sharp bound found by Andréasson [5],

$$
\frac{r_{0}}{m} \geq \frac{1}{\left(\frac{1}{3}+\sqrt{\frac{1}{9}+\frac{q^{2}}{3 r_{0}^{2}}}\right)^{2}},
$$

where $m$ and $q$ are respectively the mass and the charge of the star. This constraint is found considering regular solutions, and the upper bound is met considering infinitely thin shells, and, moreover, the condition $r_{0}^{2} / q^{2} \geq 1$ must be satisfied. We see that the limit equation (21) saturates the bound equation (22), i.e., the electrical interior Schwarzschild (in other words, the infinite pressure) limit of the Guilfoyle solution saturates the Buchdahl-Andréasson bound.

The corresponding values for $r_{0} / m$ of equation (21) as a function of $q / r_{0}$ are shown in figure 1. Note that the limit of zero charge $q / r_{0}=0$ gives $r_{0} / m=9 / 4$, the Schwarzschild result for uncharged stars [1] that saturates the Buchdahl bound [2]. The limit $q / r_{0}=1$ gives $r_{0} / m=1$, i.e., the quasiblack hole limit, the configuration where the star radius $r_{0}$ touches the horizon radius $r_{+}, r_{0}=r_{+}=m=q$ in this case [9].

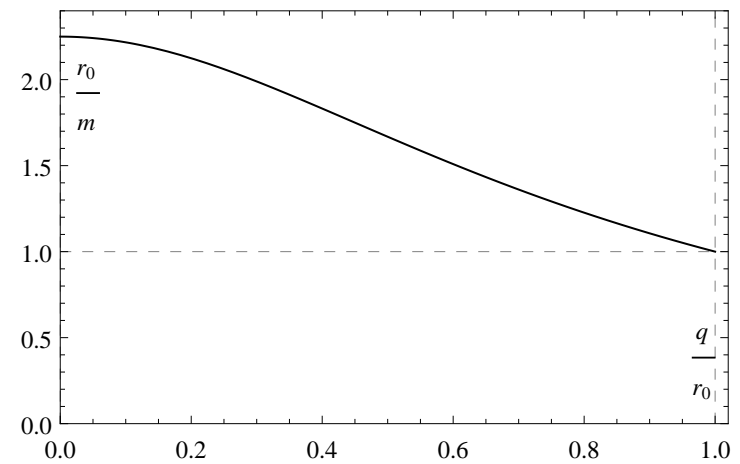

Figure 1. The line represents the star radius to mass ratio as a function of the charge to star radius ratio in the electric interior Schwarzschild limit. For each value of the charge ratio $q / r_{0}$ it corresponds an $r_{0} / m$ which is precisely the electric interior Schwarzschild limit for that electric charge. The limit saturates the BuchdahlAndréasson bound. For $q / r_{0}=0$ one finds the Schwarzschild result $r_{0} / m=9 / 4$ that saturates the Buchdahl bound, for $q / r_{0}=1$ one finds $r_{0} / m=1$, i.e., a quasiblack hole configuration. 
It would be interesting to find the Buchdahl-Andréasson bound, equation (22), as a function of $q / \mathrm{m}$ instead of $q / r_{0}$ as it is easier to understand the approach to extremality with the ratio $q / m$. Note that the electric Schwarzschild limit, equation (21), can be manipulated to give a cubic equation for the bound

$$
\left(\frac{r_{0}}{m}\right)^{3}-\frac{9}{4}\left(\frac{r_{0}}{m}\right)^{2}+\frac{3}{2}\left(\frac{q}{m}\right)^{2}\left(\frac{r_{0}}{m}\right)-\frac{1}{4}\left(\frac{q}{m}\right)^{4} \geq 0 .
$$

Unfortunately this is a nasty cubic equation. Nevertheless, one can immediately see from equation (23) that for $q / m=0$ the bound is the Buchdahl bound [1], $r_{0} / m \geq 9 / 4$, and for the extremal limit $q / m=1$ the bound is $r_{0} / m \geq 1$, i.e., the quasiblack hole limit [9]. The solution of equation (23) can be put in a non-illuminating form, namely, $\frac{r_{0}}{m} \geq \frac{3}{4}+\frac{1}{4}\left(9-\frac{8 q^{2}}{m^{2}}\right)\left[27-\frac{4 q^{2}}{m^{2}}\left(9-\frac{2 q^{2}}{m^{2}}-\frac{2 q}{m} \sqrt{\frac{q^{2}}{m^{2}}-1}\right)\right]^{-1 / 3}+$ $\frac{1}{4}\left[27-\frac{4 q^{2}}{m^{2}}\left(9-\frac{2 q^{2}}{m^{2}}-\frac{2 q}{m} \sqrt{\frac{q^{2}}{m^{2}}-1}\right)\right]^{1 / 3}$, where since $\frac{q^{2}}{m^{2}}-1$ is negative one has to deal with complex numbers. There are three real solutions, one should take the branch that interpolates between the values $9 / 4$ and 1 . More descriptive, in figure 2 the bound is displayed for $r_{0} / m$ as a function of $q / m$. The Buchdal [2] and the quasiblack hole [9] limits stand out clearly.

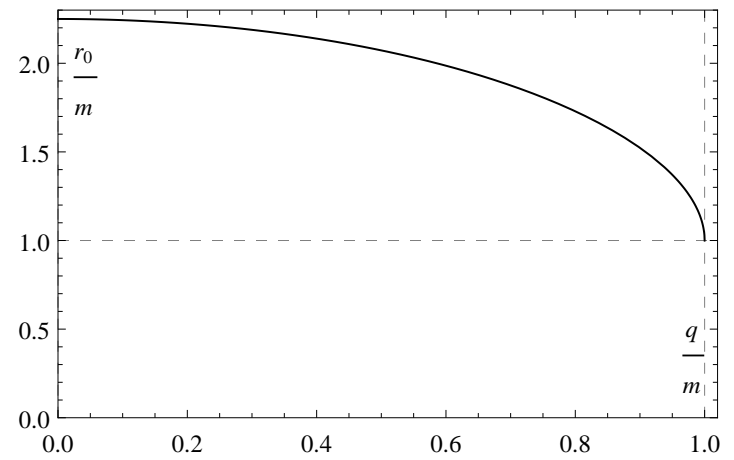

Figure 2. The line represents the star radius to mass ratio as a function of the charge to star mass ratio in the electric interior Schwarzschild limit. For each value of the charge ratio $q / m$ it corresponds an $r_{0} / m$ which is precisely the electric interior Schwarzschild limit for that electric charge. The limit saturates the BuchdahlAndréasson bound. For $q / m=0$ one finds the Schwarzschild result $r_{0} / m=9 / 4$ that saturates the Buchdahl bound, for $q / m=1$ one finds $r_{0} / m=1$, i.e., a quasiblack hole configuration.

Finally we compare the line $r_{0} / m$ of the Buchdahl-Andréasson bound as a function of $q / m$ given through equation (23) with the gravitational radius line $r_{+} / m$ as a function of $q / m$,

$$
\frac{r_{+}}{m}=1+\sqrt{1-\frac{q^{2}}{m^{2}}}
$$

see equation (41). The comparison of the two lines is done neatly in figure 3, It is clear 
from the figure that the star radius $r_{0} / m$ is always above the star's gravitational radius $r_{+} / m$, except for the quasiblack hole configuration where the two radii are coincident.

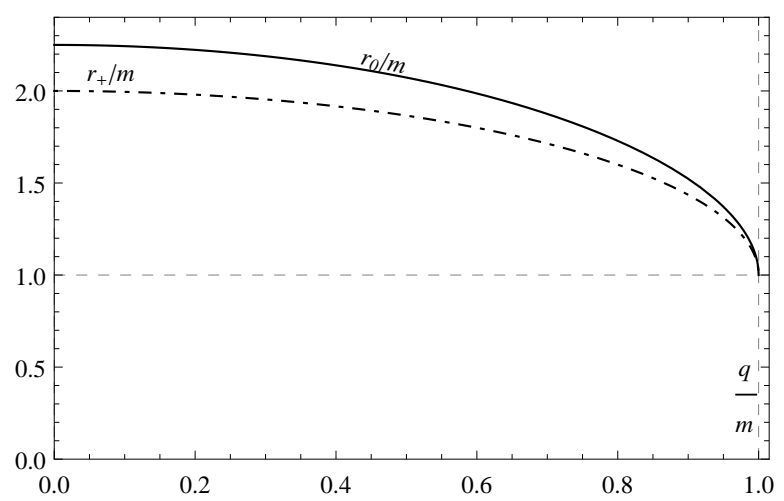

Figure 3. The solid line is the same as in figure 2. The dot-dashed line represents the gravitational radius to mass ratio as a function of the charge to star mass ratio. For $q / m=0$ one finds $r_{0} / m=9 / 4=2.25$ and the Schwarzschild radius $r_{+} / m=2$, for $q / m=1$ one finds that both radii are equal, $r_{0} / m=1$ and $r_{+} / m=1$, i.e., a quasiblack hole configuration.

\section{Conclusions}

We have shown that in addition to the electrically charged thin shells with $p+2 p_{T}=$ $\rho_{\mathrm{m}}$, the infinite central pressure Guilfoyle's stars also obey the Buchdahl-Andréasson bound, $r_{0} / m \geq 9 /\left(1+\sqrt{1+3 q^{2} / r_{0}^{2}}\right)^{2}$. It would be interesting to find a proof in Buchdahl's manner such that these infinite central pressure configurations are the limiting configurations of the bound.

\section{Acknowledgments}

JPSL thanks the Fundação para a Ciência e a Tecnologia of Portugal - FCT for support, Project No. PEst-OE/FIS/UI0099/2014. VTZ would like to thank Conselho Nacional de Desenvolvimento Científico e Tecnológico - CNPq, Brazil, for grants, and Fundação de Amparo à Pesquisa do Estado de São Paulo for a grant, Project No. 2012/08041-5. JPSL and VTZ thank Coordenação de Aperfeiçoamento do Pessoal de Nível Superior - CAPES, Brazil, for support within the Programa CSF-PVE, Project No. 88887.068694/2014-00. 


\section{References}

[1] K. Schwarzschild, "Über das Gravitationsfeld eines Kugel aus inkompressibler Flüssigkeit nach der Einsteinschen Theorie", Sitzungsberichte der Königlich Preussischen Akademie der Wissenschaften (phys.-math. Klasse, Berlin), 424 (1916); K. Schwarzschild, "On the gravitational field of a sphere of incompressible fluid according to Einstein's theory", arXiv:physics/9912033 (translation by S. Antoci).

[2] H. A. Buchdahl, "General relativistic fluid spheres", Phys. Rev. 116, 1027 (1959).

[3] H. Andréasson, "Sharp bounds on $2 m / r$ of general spherically symmetric static objects". J. Diff. Eqs. 245, 2243 (2008); arXiv:gr-qc/0702137.

[4] P. Karageorgis, J. Stalker, "Sharp bounds on $2 m / r$ for static spherical objects". Class. Quantum. Grav. 25, 195021 (2008); arXiv:0707.3632 [gr-qc].

[5] H. Andréasson, "Sharp bounds on the critical stability radius for relativistic charged spheres", Commun. Math. Phys. 288, 715 (2009); arXiv:0804.1882 [gr-qc].

[6] F. I. Cooperstock and V. de la Cruz, "Sources for the Reissner-Nordström metric", Gen. Relativ. Gravit. 9, 835 (1978).

[7] P. S. Florides, "The complete field of charged perfect fluid spheres and of other static spherically symmetric charged distributions", J. Phys. A 16, 1419 (1983).

[8] B. S. Guilfoyle, "Interior Weyl-type solutions to the Einstein-Maxwell field equations", Gen. Relativ. Gravit. 31, 1645 (1999); arXiv:gr-qc/9906089.

[9] J. P. S. Lemos and V. T. Zanchin, "Quasiblack holes with pressure: relativistic charged spheres as the frozen stars", Phys. Rev. D 81, 124016 (2010); arXiv:1004.3574 [gr-qc].

[10] J. D. V. Arbañil, J. P. S. Lemos, and V. T. Zanchin, "Incompressible relativistic spheres: Electrically charged stars, compactness bounds, and quasiblack hole configurations", Phys. Rev. D 89, 104054 (2014); arXiv:1404.7177 [gr-qc].

[11] J. P. S. Lemos, F. J. Lopes, G. M. Quinta, and V. T. Zanchin, "Compact stars with a small electric charge: the limiting radius to mass relation and the maximum mass for incompressible matter", Europ. Phys. J. C 12, 3456 (2015); arXiv:1408.1400 [gr-qc]. 